\title{
A prospective nationwide study on the impact of the level of sedation on cannulation success and complications of endoscopic retrograde cholangiopancreatography
}

\section{Bengt Hallerbäck ${ }^{a}$, Lars Enochsson ${ }^{b}$}

Northern Alvsborg Hospital, Trollhättan; Umeå University, Sunderby Research Unit, Umeå, Sweden

\begin{abstract}
Background Difficult or unsuccessful cannulation of the papilla of Vater is associated with complications during endoscopic retrograde cholangiopancreatography (ERCP). The aim of this study was to investigate whether deep sedation facilitates the cannulation and reduces the complication rate.

Methods Nationwide data from ERCP procedures were registered prospectively in the web-based Swedish Registry for Gallstone Surgery and ERCP (GallRiks). These data were used for a casecontrol study comparing the outcomes when using propofol sedation (PS) or basic sedation (BS) with midazolam in combination with opioids.

Results We analyzed 31,001 ERCP procedures in patients who had no previous ERCP. Of these, 14,907 were performed using PS and 16,094 using BS. The cannulation rate was higher in the PS group than the BS group: $89.0 \%$ vs. $86.7 \%, \mathrm{P}<0.0001$. The procedure time was longer in the PS group than in the $\mathrm{BS}$ group: 35.7 vs. $31.2 \mathrm{~min}, \mathrm{P}<0.0001$. The rate of intra-procedural complications was lower in the PS group than in the BS group: $2.9 \%$ vs. $3.7 \%, \mathrm{P}<0.0001$. The total frequency of post-procedural complications was $13.0 \%$ in the PS and $12.6 \%$ in the BS group $(\mathrm{P}=0.2607)$. The frequency of postERCP pancreatitis (PEP) was higher in the PS group than in the BS group: $4.6 \%$ vs. $4.0 \%, \mathrm{P}=0.0136$.

Conclusions PS in ERCP leads to a significantly higher cannulation success rate and fewer intra-procedural complications, but there was no significant difference in total post-ERCP complications. The procedure time was longer, and PEP was more common in the PS group. A plausible explanation could be that deep sedation might lead to more aggressive attempts to cannulate the papilla, resulting in a higher risk for PEP.
\end{abstract}

Keywords Endoscopic retrograde cholangiopancreatography, sedation, cannulation, post-ERCP pancreatitis

Ann Gastroenterol 2020; 33 (3): 1-6

Department a Surgery, Northern Alvsborg Hospital, Trollhättan (Bengt Hallerbäck); 'Surgical and Perioperative Sciences, Umeå University,

Sunderby Research Unit, Umeå (Lars Enochsson), Sweden

Conflict of Interest: None

Correspondence to: Bengt Hallerbäck, Dept of Surgery, NÄL, 46184 Trollhättan Sweden, e-mail: bengt.hallerback@vgregion.se

Financial support: This research was supported by the Local Research and Development Council, Fyrbodal, Northern Alvsborg County, Sweden, Grant nr VGFOUFBD-521861, www.fou.nu/is/fyrbodal

Received 8 December 2019; accepted 31 January 2020;

published online 13 April 2020

DOI: https://doi.org/10.20524/aog.2020.0480

\section{Introduction}

Endoscopic retrograde cholangiopancreatography (ERCP) was introduced more than 50 years ago $[1,2]$ and has become increasingly common. It is currently an almost exclusively therapeutic procedure that has progressively evolved from its previous diagnostic role. It is now often considered to be the method of choice when dealing with common bile duct stones, neoplasms of the biliary tract, and management of postoperative bile leakage. Although it is a non-surgical procedure, it is still invasive and is associated with several intra- and post-procedural complications, such as perforation, bleeding, cholangitis, and post-ERCP pancreatitis (PEP) [3-6]. The complications range from mild and transient to severe and life-threatening. Considerable variations exist in the reported 
complication rates, due to differences in the definition of complications, study design and the heterogeneity of the patient populations [7-11].

The risk of adverse events is strongly associated with the success of bile duct cannulation. Factors that increase the risk of serious complications include repeated attempts to cannulate, prolonged time for cannulation, and non-successful cannulation [7-13]. Thus, it is important to optimize the circumstances under which the endoscopist can deliver atraumatic and deep cannulation. The level of patient sedation is one such factor. A poorly sedated patient can be aware and experience pain and discomfort. The patient might move or breathe irregularly during the procedure, thus making the cannulation more difficult. The patient's discomfort might also distract the endoscopist, resulting in repeated or prolonged attempts or failure to cannulate. Thus, we were curious as to whether the level of sedation would influence cannulation success.

In general, 3 different levels of sedation are used when performing ERCP: basic sedation (BS), using intravenous (i.v.) midazolam in combination with opioids; advanced sedation, using an i.v. infusion of propofol (PS); and general anesthesia. In Sweden, general anesthesia is mostly used in highly specialized centers and when treating high-risk and complicated patients.

The aims of this study were 2 -fold. First, we studied whether there was a difference in successful cannulation frequency of the bile ducts using PS vs. BS. Second, we investigated whether the level of sedation influenced the risk for intra- and postprocedural complications.

\section{Patients and methods}

\section{Study design}

Data from ERCP procedures have been prospectively registered in the Swedish Registry for Gallstone Surgery and ERCP (GallRiks) since the registry was established on May 1, 2005 [14]. All cases of ERCP procedures registered from January 2006 to December 2016 were collected and analyzed. The outcomes were cannulation success, and intra- and postprocedural complications.

\section{Ethical consideration}

Permission to conduct the study was given by the Ethics Committee in Gothenburg (Dnr 273-15, Amendm. Dnr T559-17).

\section{The GallRiks registry}

GallRiks is a nationwide quality registry for cholecystectomy and ERCP. It is the first registry of its kind in the world and aims to provide continuously updated results on indications for both gallbladder surgery and ERCP, as well as their complications and patient satisfaction rates $[14,15]$. Mandatory data for ERCP registration include patient characteristics, indication for ERCP, admission mode, type of patient sedation/anesthesia, and details of the ERCP procedure (method of cannulation, cannulation success, procedure time, diagnostic findings, and therapeutic measures). The endoscopist registers data prospectively, immediately after the procedure, and completes an online protocol. Outcomes and possible complications after the ERCP procedures are collected via a review of the patient's medical chart after 30 days. This is done by an appointed coordinator who registers each outcome in an online follow-up questionnaire in the same database.

To document the coverage of the GallRiks registry, a comparison between the data registered in GallRiks and data found in the Swedish National Patient Register-where all ICD diagnostic codes and surgical intervention codes are registered-is performed annually. These comparisons regularly show that approximately $90 \%$ of all ERCP procedures annually performed in Sweden are registered in GallRiks [14,15]. To further document the validity of GallRiks, an independent audit group regularly studied the medical records and compared each with GallRiks data in participating hospitals once every 3 years. The results showed a complete match between the database and corresponding medical records in $97.3 \%$ of ERCP cases $[14,16]$.

\section{Definition of variables registered in GallRiks}

\section{Type of sedation}

- Basic sedation: i.e., conscious sedation utilizing i.v. midazolam in combination with opioids.

- Advanced sedation: i.e., unconscious sedation utilizing i.v. infusion of propofol.

\section{Procedure time}

Time elapsed from the start of endoscope introduction until the endoscope is withdrawn.

\section{Deep cannulation of bile ducts}

Filling the bile duct with contrast using a guidewire or catheter introduced into the duct.

\section{Intraprocedural complications}

Bleeding, extravasation of contrast, perforation or other reasons resulting in premature interruption of the procedure.

\section{Intraprocedural bleeding}

Bleeding from the papilla needing acute treatment with blood transfusion or operation.

\section{Intraprocedural extravasation of contrast}

Contrast leakage from the bile duct into the retroperitoneal space. 


\section{Post-ERCP complication}

Any unfavorable event registered within 30 days after the procedure and leading to hospitalization, operation, blood transfusion, antibiotic treatment or other measure.

\section{PEP}

The definition of PEP was in accordance with the classification by Cotton et al [9], i.e., abdominal pain associated with serum amylase at least 3 times the normal level at $24 \mathrm{~h}$ or more after the ERCP procedure.

\section{Non-index ERCP procedure}

Any ERCP procedure registered in the registry as secondary to another intervention will not be followed in accordance with the 30-day control schedule; thus, it is defined as a non-index procedure.

\section{Selection of ERCP procedures to be included in the study}

Procedures categorized as non-index ERCP and cases that did not complete 30 days of follow up were excluded, as were ERCP procedures with the following indications: acute and chronic pancreatitis, primary sclerosing cholangitis, scheduled controls, and stent dysfunction. Cases with previous endoscopic sphincterotomy, procedures not aiming for bile duct cannulation, procedures with missing data regarding sedation method, and cases performed under general anesthesia were also excluded.

\section{Statistical analysis}

Pearson's chi-square test was used to evaluate the hypotheses of the variables in contingency tables. A P-value of $<0.05$ was considered statistically significant. Univariate and multivariate logistic regression analyses of the effects of propofol and basic sedation on the outcome were undertaken. In the multivariate logistic regression analyses, adjustments were made for sex, age, American Society of Anesthesiology (ASA) class (ASA I-II vs. ASA >II), and indications for the ERCP. The effects of the analyzed variables are presented as odds ratios (OR) with 95\% confidence intervals (CI). All analyses were carried out using JMP Pro 14.2.0 (64-bit) (SAS Institute Inc., Cary, North Carolina, USA) for Windows 10 Education, version 1803 (Microsoft Corporation, USA).

\section{Results}

The registry facilitated 78,429 ERCP cases from 1st January, 2006, to 31st December, 2016. After exclusion of cases not eligible to participate in the analysis, we had 31,001 cases, of which 16,094 (51.9\%) underwent ERCP under BS and 14,907 (48.1\%) under PS (Fig. 1).

\section{Patient characteristics}

There was a slight predominance of females in both groups (Table 1). The ASA classification showed a significant difference, with $70.4 \%$ of the cases in the BS group classified as ASA I-II vs. $66.7 \%$ in the PS group $(\mathrm{P}<0.0001)$. There was also a small but significant difference in age between the groups, with a mean age in the BS group of 71.0 years, compared with 71.7 years in the PS group $(\mathrm{P}=0.0001)$. Indications for the ERCP procedures differed significantly between the 2 groups $(\mathrm{P}<0.0001)$ (Table 2$)$.

Table 1 Demographics of the cohort of 30,001 patients who underwent ERCP under basic or propofol sedation from January 2006 to December 2016

\begin{tabular}{|c|c|c|c|c|c|c|}
\hline \multicolumn{2}{|c|}{ Characteristics } & \multirow{2}{*}{\multicolumn{2}{|c|}{$\begin{array}{c}\text { Basic sedation } \\
n=16,094\end{array}$}} & \multirow{2}{*}{\multicolumn{2}{|c|}{$\begin{array}{l}\text { Propofol } \\
n=14,907\end{array}$}} & \multirow[t]{3}{*}{ P-value } \\
\hline & & & & & & \\
\hline & & $\mathrm{n}$ & $\%$ & $\mathrm{n}$ & $\%$ & \\
\hline \multirow[t]{5}{*}{ Sex } & Female & 8656 & 53.8 & 8187 & 54.9 & 0.0447 \\
\hline & Male & 7438 & 46.2 & 6720 & 45.1 & \\
\hline & ASA $1-2$ & 11330 & 70.4 & 9944 & 66.7 & $<0.0001$ \\
\hline & & Mean & SEM & Mean & SEM & P\# \\
\hline & Age & 71.0 & 0.1 & 71.7 & 0.1 & 0.0001 \\
\hline
\end{tabular}

$\mathrm{P}^{*}$ : Pearson chi-square test; $\mathrm{P} \#$ : Student's t-test

ERCP, endoscopic retrograde cholangiopancreatography; ASA, American Society of Anesthesiology; SEM, standard error of the mean

Table 2 Indications for ERCP procedure in the cohort of 30,001 patients who underwent ERCP in basic or propofol sedation from January 2006 to December 2016

\begin{tabular}{|c|c|c|c|c|c|}
\hline \multirow[t]{2}{*}{ Indications } & \multicolumn{2}{|c|}{ Basic sedation } & \multicolumn{2}{|c|}{ Propofol } & \multirow[t]{2}{*}{ P-value ${ }^{*}$} \\
\hline & $\mathrm{n}$ & $\%$ & $\mathrm{n}$ & $\%$ & \\
\hline CBDS & 5972 & 37.1 & 5840 & 39.2 & $<0.0001$ \\
\hline Obstructive jaundice & 5304 & 33.0 & 4234 & 28.4 & \\
\hline Malignancy & 2341 & 14.6 & 2225 & 14.9 & \\
\hline Cholangitis & 1453 & 9.0 & 1524 & 10.2 & \\
\hline Other indications & 584 & 3.6 & 574 & 3.9 & \\
\hline $\begin{array}{l}\text { Gallstone pancreatitis } \\
\text { prophylaxis }\end{array}$ & 224 & 1.4 & 324 & 2.2 & \\
\hline $\begin{array}{l}\text { Susp. Post op bile } \\
\text { leakage }\end{array}$ & 213 & 1.3 & 171 & 1.1 & \\
\hline $\begin{array}{l}\text { Intraop diagnostic } \\
\text { ERCP }\end{array}$ & 3 & 0.0 & 15 & 0.1 & \\
\hline
\end{tabular}




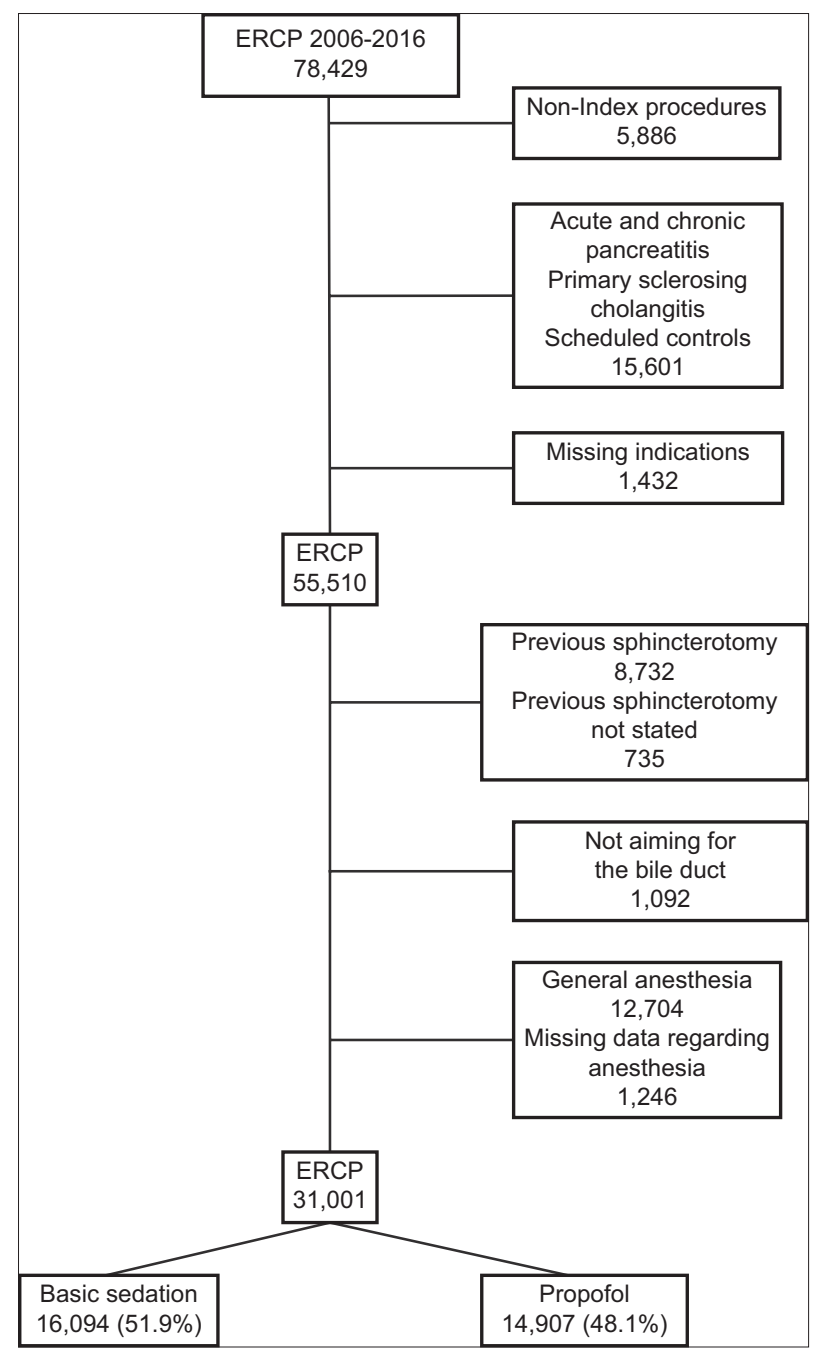

Figure 1 Cohort selection of endoscopic retrograde cholangiopancreatography (ERCP) procedures from the GallRiks database

\section{Common bile duct cannulation, procedure time, and complications}

Successful deep cannulation of the common bile duct was frequent in both groups, but showed a significantly higher success rate in the PS group vs. the BS group: $89.0 \%$ vs. $86.8 \%$, $\mathrm{P}<0.0001$ (Table 3). The procedure time was longer in the PS group than the BS group: mean 35.7 (SEM 0.1) vs. 31.2 (SEM $0.2)$ min (Table 3).

Intraprocedural complications were significantly more common in the BS group than in the PS group: $3.7 \%$ vs. $2.9 \%$, $\mathrm{P}<0.0001$ (Table 3 ). The rate of intra-procedural bleeding was $1.1 \%$ in $\mathrm{BS}$ vs. $0.6 \%$ in the PS group $(\mathrm{P}<0.0001)$. In contrast, there was no significant difference between the groups in the total frequency of postprocedural complications: $13.0 \%$ in the PS vs. $12.6 \%$ in the BS group ( $\mathrm{P}=0.2607)$.

PEP, however, was significantly more common in the PS group than in the BS group: $4.6 \%$ vs. $4.0 \%, \mathrm{P}=0.0136$. Postoperatively detected perforation of the gut was also more common in the PS group than in the BS group ( $0.5 \%$ vs. $0.4 \%$, $\mathrm{P}=0.0385$ ).

Cannulation success, as well as intra- and postprocedural complications, were also analyzed in uni- and multivariate analyses after adjustment for age, sex, ASA classification and indication for ERCP. Table 4 shows that these analyses did not alter the outcome. The OR for successful deep bile duct cannulation was higher, and the intraprocedural complication rate was lower in patients in the PS group vs. the BS patients. There was no significant difference in OR for total postprocedural complications but both PEP (OR 1.16, 95\%CI 1.04-1.29) and perforation of the gut (OR 1.42, 95\%CI 1.02-1.99) were still more common in the PS group than in the BS group.

\section{Discussion}

Our results demonstrate that the level of patient sedation does influence outcomes in ERCP. Successful deep cannulation of the bile duct is more common with propofol. This is in accordance with our theory that an unconscious patient-still, calm, and with regular breathing movementsmakes it easier for the endoscopist to cannulate into the deep bile duct. The specific moment of cannulation is one of the most crucial factors influencing the risk for intraand postprocedural complications [7-11,17]; thus, it would be logical to find a lower incidence of complications in the cohort of patients sedated with propofol. The PS group also had fewer intraprocedural complications, including intraprocedural bleeding. This result did not change when multivariate analyses were performed and adjusted for age, sex, ASA classification and indication.

Importantly, our analyses did not demonstrate a lower frequency of postprocedural complications in the PS group. No significant differences in total postprocedural complications were seen in the general analysis or multivariate analyses.

However, we did find a higher frequency of PEP in the PS group. This is seemingly a paradox and could eventually be explained if the PS group consisted of more subjects with an enhanced risk for PEP, including younger women and more critically ill patients with higher ASA classification. The multivariate analysis, however, did not suggest that the PEP risk was due to age, sex, ASA classification, or indication for ERCP (Table 3).

The procedure time was significantly longer in the PS group. This is also somewhat unexpected since, in theory, it should be possible to perform the procedure in a shorter time in a deeply sedated patient lying still and unaffected. The reason for the longer procedure duration in the PS group is obscure, but it might be that, in the effort to achieve successful cannulation in difficult cases, the endoscopist is likely to continue trying in propofol-sedated patients, which can also enhance the risk of PEP. This could be a conceivable explanation for the higher proportion of PEP in the PS group, although the successful cannulation rate was higher. The higher frequency of perforations might also be due to more aggressive attempts to cannulate in deeply sedated patients. 
Table 3 Cannulation success, intra- and postprocedural complications detected, and ERCP procedure time among 30,001 patients undergoing ERCP under basic sedation ( $\mathrm{n}=16,094)$ or propofol sedation $(\mathrm{n}=14,907)$

\begin{tabular}{|c|c|c|c|c|c|c|}
\hline \multirow[t]{3}{*}{ Complications } & & \multirow{2}{*}{\multicolumn{2}{|c|}{$\begin{array}{l}\text { Basic sedation } \\
\qquad n=16,094\end{array}$}} & \multirow{2}{*}{\multicolumn{2}{|c|}{$\begin{array}{l}\text { Propofol } \\
n=14,907\end{array}$}} & \multirow[t]{3}{*}{ P-value ${ }^{*}$} \\
\hline & & & & & & \\
\hline & & $\mathrm{n}$ & $\%$ & $\mathrm{n}$ & $\%$ & \\
\hline & Deep cannulation of bile duct & 13958 & 86.7 & 13270 & 89.0 & $<0.0001$ \\
\hline \multirow[t]{3}{*}{ Intraprocedural complications } & Intraprocedural complications & 588 & 3.7 & 425 & 2.9 & $<0.0001$ \\
\hline & Intraprocedural bleeding & 180 & 1.1 & 94 & 0.6 & $<0.0001$ \\
\hline & Extravasation of contrast & 154 & 1.0 & 144 & 1.0 & 0.9345 \\
\hline \multirow[t]{10}{*}{$\begin{array}{l}\text { Complications within } 30 \text { days post- } \\
\text { procedure }\end{array}$} & Post-ERCP complications & 2030 & 12.6 & 1944 & 13.0 & 0.2607 \\
\hline & ERCP bleeding & 212 & 1.3 & 184 & 1.2 & 0.5159 \\
\hline & Pancreatitits & 649 & 4.0 & 686 & 4.6 & 0.0136 \\
\hline & Perforation of the gut & 60 & 0.4 & 79 & 0.5 & 0.0385 \\
\hline & Cholangitis & 357 & 2.2 & 331 & 2.2 & 0.9894 \\
\hline & Mortality $<30$ days & 1154 & 7.2 & 1033 & 6.9 & 0.4082 \\
\hline & & \multicolumn{2}{|c|}{ Basic sedation } & \multicolumn{2}{|c|}{ Propofol } & P-value\# \\
\hline & & \multicolumn{2}{|c|}{$\mathrm{n}=16,094$} & \multicolumn{2}{|c|}{$\mathrm{n}=14,907$} & \\
\hline & & Mean & SEM & Mean & SEM & \\
\hline & ERCP procedure time (min) & 31.2 & 0.1 & 35.7 & 0.2 & $<0.0001$ \\
\hline
\end{tabular}

$\mathrm{P}^{*}$ : Pearson chi-square test; $\mathrm{P} \#$ : Student's t-test

ERCP, endoscopic retrograde cholangiopancreatography; SEM, standard error of the mean

Table 4 Intra- and postprocedural complications among 30,001 patients undergoing ERCP.Uni- and multivariate analysis of propofol sedation $(\mathrm{n}=14,907)$ vs. basic sedation $(\mathrm{n}=16,094)$

\begin{tabular}{|c|c|c|c|c|c|c|}
\hline \multirow[t]{3}{*}{ Complications } & & \multicolumn{4}{|c|}{ Propofol vs. basic sedation } & \multirow[t]{3}{*}{ P-value } \\
\hline & & \multicolumn{2}{|c|}{ Univariate analysis } & \multicolumn{2}{|c|}{$\begin{array}{l}\text { Multivariate } \\
\text { analysis }^{\star}\end{array}$} & \\
\hline & & OR & $95 \% \mathrm{CI}$ & OR & $95 \% \mathrm{CI}$ & \\
\hline & Deep cannulation of bile duct & 1.23 & $1.16-1.33$ & 1.23 & $1.14-1.31$ & $<0.0001$ \\
\hline \multirow[t]{3}{*}{ Intraprocedural complications } & Intraprocedural complications & 0.77 & $0.68-0.88$ & 0.77 & $0.68-0.87$ & $<0.0001$ \\
\hline & Intraprocedural bleeding & 0.56 & $0.44-0.72$ & 0.55 & $0.43-0.71$ & $<0.0001$ \\
\hline & Extravasation of contrast & 1.00 & $0.80-1.27$ & 1.03 & $0.82-1.30$ & 0.7815 \\
\hline \multirow[t]{6}{*}{ Complications within 30 days post-procedure } & Post-ERCP complications & 1.04 & $0.97-1.11$ & 1.05 & $0.98-1.12$ & 0.1725 \\
\hline & ERCP bleeding & 0.94 & $0.77-1.14$ & 0.90 & $0.74-1.10$ & 0.3163 \\
\hline & Pancreatitis & 1.15 & $1.03-1.28$ & 1.16 & $1.04-1.29$ & 0.0098 \\
\hline & Perforation of the gut & 1.42 & $1.02-1.99$ & 1.42 & $1.02-1.99$ & 0.0393 \\
\hline & Cholangitis & 1.00 & $0.86-1.16$ & 1.01 & $0.87-1.17$ & 0.9229 \\
\hline & Mortality $<30$ days & 0.96 & $0.88-1.05$ & 0.95 & $0.87-1.05$ & 0.3309 \\
\hline
\end{tabular}

${ }^{*}$ Adjusted for age, sex, American Society of Anesthesiologists (ASA) classification and indication Propofol vs Basic sedation, Multivariate analysis

ERCP, endoscopic retrograde cholangiopancreatography; OR, odds ratio; CI, confidence interval; SEM, standard error of the mean 
The strength of this large nationwide prospective registry study is that it reflects the general outcomes in the treatment of this category of patients. One possible weakness of the study is that a registry of this size must be easy to use and administer in general practice; thus, it is difficult to focus on certain specific parameters, such as the number of attempts at cannulation, the number and depth of eventual unintended pancreatic cannulations, and the types of device used. It is not possible to record this level of detail in a nationwide registry. Another possible weakness in the study is the differences in ERCP indications, as seen in Table 2. It can be argued that different indications might affect the choice of sedation. However, it is unlikely that the indication alone would be a significant predictor of the complexity of the ERCP procedure, since all procedures in our study were performed in patients who had undergone no previous ERCP attempt. Furthermore, the database can provide no information about the endoscopist's expectation regarding the complexity of the procedure. In Sweden, patients with an expected high risk for complex cannulation and complication are mostly referred to special centers, where the procedure is often performed under general anesthesia, and such cases were not included in this study.

We conclude that propofol sedation in ERCP patients leads to a higher frequency of successful cannulation of the bile duct and a lower frequency of intraprocedural complications. The total frequency of postprocedural complications is not affected by the choice of sedation. However, it is possible that sedation with propofol might lead to longer procedure times and more aggressive attempts to cannulate the papilla; this in turn might lead to a higher risk for PEP and perforation.

\section{Summary Box}

\section{What is already known:}

- Difficult or unsuccessful cannulation of the papilla of Vater is associated with complications when performing endoscopic retrograde cholangiopancreatography (ERCP)

\section{What the new findings are:}

- Propofol sedation leads to a higher frequency of successful cannulation of the bile duct

- Propofol sedation leads to a lower frequency of intraprocedural complication

- Propofol sedation leads to a higher frequency of post-ERCP pancreatitis which might be due to more aggressive attempts to cannulate in difficult cases

\section{References}

1. Cotton PB. Cannulation of the papilla of Vater by endoscopy and retrograde cholangiopancreatography (ERCP). Gut 1972;13:1014-1025.

2. Cotton PB, Blumgart LH, Davies GT, et al. Cannulation of papilla of Vater via fiber-duodenoscope. Assessment of retrograde cholangiopancreatography in 60 patients. Lancet 1972;1:53-58.

3. Anderson MA, Fisher L, Jain R, et al; ASGE Standards of Practice Committee. Complications of ERCP. Gastrointest Endosc 2012;75:467-473.

4. Adler DG, Baron TH, Davila RE, et al; Standards of Practice Committee of American Society for Gastrointestinal Endoscopy. ASGE guideline: the role of ERCP in diseases of the biliary tract and the pancreas. Gastrointest Endosc 2005;62:1-8.

5. Chandrasekhara V, Khashab MA, Muthusamy VR, et al; ASGE Standards of Practice Committee. Adverse events associated with ERCP. Gastrointest Endosc 2017;85:32-47.

6. Arata S, Takada T, Hirata K, et al. Post-ERCP pancreatitis. J Hepatobiliary Pancreat Sci 2010;17:70-78.

7. Bailey AA, Bourke MJ, Williams SJ, et al. A prospective randomized trial of cannulation technique in ERCP: effects on technical success and post-ERCP pancreatitis. Endoscopy 2008;40:296-301.

8. Freeman ML, DiSario JA, Nelson DB, et al. Risk factors for postERCP pancreatitis: a prospective, multicenter study. Gastrointest Endosc 2001;54:425-434.

9. Cotton PB, Lehman G, Vennes J, et al. Endoscopic sphincterotomy complications and their management: an attempt at consensus. Gastrointest Endosc 1991;37:383-393.

10. Testoni PA, Mariani A, Giussani A, et al; SEIFRED Group. Risk factors for post-ERCP pancreatitis in high- and low-volume centers and among expert and non-expert operators: a prospective multicenter study. Am J Gastroenterol 2010;105:1753-1761.

11. Cheon YK, Cho KB, Watkins JL, et al. Frequency and severity of post-ERCP pancreatitis correlated with extent of pancreatic ductal opacification. Gastrointest Endosc 2010;65:385-393.

12. Andriulli A, Loperfido S, Napolitano G, et al. Incidence rates of post-ERCP complications: a systematic survey of prospective studies. Am J Gastroenterol 2007;102:1781-1788.

13. Cheng CL, Sherman S, Watkins JL, et al. Risk factors for post-ERCP pancreatitis: a prospective multicenter study. Am J Gastroenterol 2006;101:139-147.

14. Enochsson L, Thulin A, Osterberg J, Sandblom G, Persson G. The Swedish Registry of Gallstone Surgery and Endoscopic Retrograde Cholangiopancreatography (GallRiks): A nationwide registry for quality assurance of gallstone surgery. JAMA Surg 2013;148:471-478.

15. Enochsson L, Swahn F, Arnelo U, Nilsson M, Löhr M, Persson G. Nationwide, population-based data from 11,074 ERCP procedures from the Swedish Registry for Gallstone Surgery and ERCP. Gastrointest Endosc 2010;72:1175-1184, 1184.e1-3.

16. Rystedt J, Montgomery A, Persson G. Completeness and correctness of cholecystectomy data in a national register-GallRiks. Scand J Surg 2014;103:237-244.

17. Swahn F, Nilsson M, Arnelo U, Löhr M, Persson G, Enochsson L. Rendezvous cannulation technique reduces post-ERCP pancreatitis: a prospective nationwide study of 12,718 ERCP procedures. Am J Gastroenterol 2013;108:552-559. 